\title{
The Maximum of a Quasi-Smooth Function
}

\author{
By J. Ernest Wilkins, Jr. and Theodore R. Hatcher
}

\begin{abstract}
Let $Z$ be the class of real-valued functions, defined and continuous on the closed interval $I=[-1,1]$, such that $f( \pm 1)=0$ and $|f(\xi)-2 f\{(\xi+\eta) / 2\}+f(\eta)| \leqslant|\xi-\eta|$ for all $\xi$ and $\eta$ in $I$. Let $K=\sup _{f \in 7} \max _{x \in I}|f(x)|$. We will prove that $13 / 10 \leqslant K \leqslant 1014 / 779<$ 1.301669 .
\end{abstract}

1. Introduction. Zygmund [7] introduced the class of real valued functions $f(x)$, defined and continuous on the closed interval $I=[-1,1]$, such that

$$
|f(\xi)-2 f\{(\xi+\eta) / 2\}+f(\eta)| \leqslant M|\xi-\eta|
$$

for some constant $M$ and all $\xi$ and $\eta$ in $I$. Timan [4], [5], [6] called such functions "quasi-smooth". Let $Z$ be the class of quasi-smooth functions for which $M=1$ and $f(-1)=f(+1)=0$. Suppose that

$$
K=\sup _{f \in Z} \max _{x \in I}|f(x)| .
$$

Timan [6] proved that $K \leqslant 4 / 3$ and asserted that this was not the best result. Brudnyi [1] attributed to Abramov the claim that $K \leqslant 383 / 288$. He exhibited a function in $Z$ whose maximum value is $5 / 4$; hence $K \geqslant 5 / 4$. Sokolova [3] proved that $K \leqslant 4 / 3-4 / 381=168 / 127<1.322835$, and conjectured that $K=5 / 4$.

In this paper we will prove that

$$
1.3=13 / 10 \leqslant K \leqslant 1014 / 779<1.301669,
$$

thus disproving Sokolova's conjecture. (In no way discouraged by her failure, we conjecture that $K=13 / 10$.)

The proof starts with the derivation in Section 2 of upper bounds for $|f(\xi)|$ as $f$ ranges over $Z$ when $\xi$ is one of twenty-six particular numbers in $I$. Each of these upper bounds is the consequence of a particular identity in the functional values of $f$ and the inequality (1). It is easy to see (cf. Section 5) that there is a function $f_{0}$ in $Z$ and a point $x_{0}$ in $I$ such that $f_{0}\left(x_{0}\right)=K$. In Sections 4 and 5 we derive two basic inequalities from which can be inferred upper bounds on $K$ if it is known that $x_{0}$ lies in a subinterval $(a, b)$ of $I$ and if upper bounds for $f(a)$ and $f(b)$ as $f$ ranges over $Z$ are known. In Section 6 we apply these basic inequalities to each of the subintervals determined by adjacent values of $\xi$ considered in Section 2, and thereby obtain the asserted upper bound on $K$. In Section 3 we show that the membership in $Z$ of a piecewise linear continuous function $f(x)$ on $I$, whose corners lie in an arithmetic progression (with distance $N^{-1}$ between successive numbers) can be decided in

Received June 24, 1982.

1980 Mathematics Subject Classification. Primary 26D20. 
$2 N(2 N-1)$ arithmetical tests, and then exhibit such a function with $N=20$ that passes all of the tests, so that it is in $Z$, and that has the value $13 / 10$ when $x=3 / 10$. This demonstrates the asserted lower bound on $K$. In Section 7 we describe the manner in which we used a computer to assist in the discovery of the functional identities used in Section 2 and of the piecewise linear function in $Z$ recorded in Section 3. We emphasize, however, that the proofs of the identities and of the properties of the piecewise linear function are independent of the computer.

2. Some Preliminary Numerical Results. We will show in this section that, if $f \in Z$,

$$
\begin{gathered}
|f(0)| \leqslant 1, \quad|f(1 / 2)| \leqslant 1, \quad|f(1 / 4)| \leqslant 5 / 4, \quad|f(1 / 3)| \leqslant 23 / 18, \\
|f(1 / 5)| \leqslant 32 / 25, \quad|f(1 / 6)| \leqslant 35 / 27, \quad|f(2 / 7)| \leqslant 127 / 98, \\
|f(2 / 9)| \leqslant 415 / 324, \quad|f(2 / 11)| \leqslant 2821 / 2178, \quad|f(3 / 10)| \leqslant 13 / 10 \\
|f(3 / 16)| \leqslant 373 / 288, \quad|f(3 / 20)| \leqslant 193 / 150, \quad|f(4 / 23)| \leqslant 686 / 529 \\
|f(5 / 17)| \leqslant 751 / 578, \quad|f(5 / 18)| \leqslant 419 / 324, \quad|f(5 / 31)| \leqslant 1244 / 961, \\
|f(5 / 32)| \leqslant 31 / 24, \quad|f(7 / 20)| \leqslant 127 / 100, \quad|f(7 / 22)| \leqslant 313 / 242, \\
|f(9 / 31)| \leqslant 161 / 124, \quad|f(11 / 35)| \leqslant 3179 / 2450, \quad|f(11 / 36)| \leqslant 421 / 324, \\
|f(13 / 42)| \leqslant 1145 / 882, \quad|f(19 / 64)| \leqslant 1331 / 1024, \quad|f(23 / 76)| \leqslant 1877 / 1444, \\
|f(25 / 84)| \leqslant 2293 / 1764 .
\end{gathered}
$$

Consider (1) when $\xi=-1, \eta=1, M=1$. Because $f( \pm 1)=0$, it follows that $|f(0)| \leqslant 1$. We infer from the identity

$$
2 f(1 / 2)=[2 f(1 / 2)-f(0)-f(1)]+f(0)+f(1),
$$

an application of (1) when $M=1$, the just established upper bound for $f(0)$, and the vanishing of $f(1)$, that $|f(1 / 2)| \leqslant 1$. Because $f(-x) \in Z$ when $f(x) \in Z$, it follows that $|f(-1 / 2)| \leqslant 1$ also.

The remaining inequalities in (2) are consequences of analogous, but more complicated identities, recorded at the end of the paper, and similar reasoning.

3. Piecewise Linear Functions in $Z$. Let $N$ be a positive integer, and $x_{k}=$ $(k-N) / N, k=0,1, \ldots, 2 N$. Let $y$ be a $2 N+1^{\prime}$ tuple $\left(y_{0}, y_{1}, \ldots, y_{2 n}\right)$ such that $y_{0}=y_{2 N}=0$, and suppose that $f(x)$ is a real-valued continuous function on $I$ such that $f\left(x_{k}\right)=y_{k}$. A necessary condition that $f \in Z$ is that

$$
\left|y_{k}-2 y_{k+l}+y_{k+2 l}\right| \leqslant 2 l / N
$$

for all integers $k$ and $l$ such that $0 \leqslant k \leqslant 2 N-2,1 \leqslant l \leqslant N-(k / 2)$. It is also necessary that (3) hold when $l$ is half of an odd integer such that $3 \leqslant 2 l \leqslant 2 N-k$, if $y_{k+l}$ is defined as $f[(k+l-N) / N]$ for such values of $l$. If $f$ is known to be linear on each interval $\left(x_{k}, x_{k+1}\right)$, the following lemma asserts that these conditions are sufficient.

LEMmA 1. Suppose that $x_{k}=(k-N) / N$, that $f(x)$ is linear on each interval $\left(x_{k}, x_{k+1}\right)$, and that $f\left(x_{k}\right)=y_{k}$. Then $f \in Z$ if, and only if, $y_{0}=y_{2 N}=0$ and (3) holds whenever $k$ and $2 l$ are integers such that $0 \leqslant k \leqslant 2 N-2,2 \leqslant 2 l \leqslant 2 N-k$, and the value $y_{k+1 / 2}$ is defined as $\left(y_{k}+y_{k+1}\right) / 2$. 
Consider the functions $Q_{j}(\xi, \eta)=(-1)^{j}\{f(\xi)-2 f[(\xi+\eta) / 2]+f(\eta)\}-\eta+\xi$ $(j=0,1)$. For each $\eta$ in $(-1,1)$ these functions are piecewise linear, continuous functions of $\xi$ on $[-1, \eta]$ with corners in the set consisting of $\eta$ and those points $x_{k}$ and $2 x_{k}-\eta$ that lie in $[-1, \eta]$. It follows that $Q_{j}(\xi, \eta) \leqslant 0$ when $-1 \leqslant \xi \leqslant \eta$ if, and only if, $Q_{j}\left(x_{k}, \eta\right) \leqslant 0$ and $Q_{j}\left(2 x_{k}-\eta, \eta\right) \leqslant 0$ for all $k$ such that $x_{k}<\eta$ and $-1 \leqslant 2 x_{k}-\eta<\eta$, respectively. (Note that $Q_{j}(\eta, \eta) \equiv 0$.) For each $j$ and $k$, the functions $Q_{j}\left(x_{k}, \eta\right)$ and $Q_{j}\left(2 x_{k}-\eta, \eta\right)$ are piecewise linear, continuous functions of $\eta$ on the respective intervals $\left[x_{k}, 1\right]$ and $\left[x_{k}, x_{k}^{\prime}\right]$, in which $x_{k}^{\prime}=\min \left(2 x_{k}+1,1\right)$. The corners of $Q_{j}\left(x_{k}, \eta\right)$ lie in the set of those points $x_{n}$ and $2 x_{n}-x_{k}$ that are in $\left[x_{k}, 1\right]$, and the corners of $Q_{j}\left(2 x_{k}-\eta, \eta\right)$ lie in the set of those points $x_{n}$ and $2 x_{k}-x_{n}$ that are in $\left[x_{k}, x_{k}^{\prime}\right]$. Moreover $Q_{j}\left(x_{k}, \eta\right) \leqslant 0$ and $Q_{j}\left(2 x_{k}-\eta, \eta\right) \leqslant 0$ for the appropriate values of $k$ and $\eta$ if, and only if, $Q_{j}\left(x_{k}, \eta_{n}\right) \leqslant 0$ and $Q_{j}\left(2 x_{k}-\eta_{n}, \eta_{n}\right) \leqslant 0$ whenever $\eta_{n}$ is an appropriate corner. Because $2 x_{h}-x_{k}=$ $x_{2 h-k}$, it is now clear that $Q_{j}(\xi, \eta) \leqslant 0$ for all $\xi$, $\eta$ such that $-1 \leqslant \xi \leqslant \eta \leqslant 1$ if, and only if, $Q_{j}\left(x_{k}, x_{h}\right) \leqslant 0$ for all $k$ and $h$ such that $0 \leqslant k<h \leqslant 2 N$. If we define $l$ so that $h=k+2 l$, then $2 l$ is a positive integer and the lemma is now obvious, after we observe that (3) is trivial when $l=1 / 2$, and that $1 / 2$ is the only permissible value of $l$ when $k=2 N-1$.

Consider the piecewise linear function $f_{1}(x)$ defined when $N=20$ by the value $y=(0,1 / 5,2 / 5,11 / 20,3 / 5,3 / 4,17 / 20,19 / 20,19 / 20,19 / 20,1,11 / 10,6 / 5,6 / 5$, $6 / 5,23 / 20,11 / 10,21 / 20,1,1,1,11 / 10,6 / 5,5 / 4,5 / 4,5 / 4,13 / 10,5 / 4,23 / 20$, $21 / 20,1,21 / 20,1,19 / 20,7 / 8,7 / 10,3 / 5,11 / 20,2 / 5,1 / 4,0)$. There are $2 N(2 N-1)=1560$ tests $(3)$ in Lemma 1 , and all of them are passed, so $f_{1}(x) \in Z$. Because $f_{1}(3 / 10)=13 / 10, f_{1}(0)=1, f_{1}(1 / 2)=1, f_{1}(1 / 4)=5 / 4$, we conclude that the inequalities in (2) for $f(3 / 10), f(0), f(1 / 2)$ and $f(1 / 4)$ are best possible, and that $K \geqslant 13 / 10$. This example refutes Sokolova's conjecture [3] that $K=3 / 4$.

It is also true that there exists, for each $\xi$ in the set $\{1 / 3,1 / 5,1 / 6,2 / 7,2 / 9$, $3 / 16,3 / 20,4 / 23,5 / 31,7 / 20,7 / 22,11 / 36,19 / 64\}$, a piecewise linear function in $Z$ for which the upper bound in (2) is attained. Because all of these upper bounds are less than 13/10, this knowledge does not help us improve the lower bound $13 / 10$ for $K$, and we omit the proof of this assertion. We have been unable to decide whether the remaining inequalities in (2) are best possible.

4. The First Basic Inequality. For each $f$ in $Z$ let $x_{f}$ be a point in $I$ at which $|f(x)|$ attains its maximum value $\alpha_{f}$. It follows from (1) that, if $\xi$ and $\eta=2 x_{f}-\xi$ are both in $I$, then

$$
2 \alpha_{f} \leqslant 2\left|x_{f}-\xi\right|+|f(\xi)|+|f(\eta)|, \quad \alpha_{f} \leqslant 2\left|x_{f}-\xi\right|+2 A(\xi),
$$

if $A(\xi)$ is an upper bound on $Z$ for $|f(\xi)| / 2$. Therefore, $\alpha_{f} \leqslant K^{\prime}$ for some $K^{\prime}$ if $\xi \in I$, $K^{\prime} \geqslant 2 A(\xi)$, and

$$
\begin{aligned}
& \max \left[-(1-\xi) / 2, \xi+A(\xi)-\left(K^{\prime} / 2\right)\right] \\
& \leqslant x_{f} \leqslant \min \left[(1+\xi) / 2, \xi-A(\xi)+\left(K^{\prime} / 2\right)\right]
\end{aligned}
$$

If $a$ and $b$ are any two points in $I$ such that $a<b$, the set of values $x_{f}$ that satisfy (5) either when $\xi=a$ or when $\xi=b$ surely contains the interval $(a, b)$ if $\max \left[-(1-b) / 2, b+A(b)-\left(K^{\prime} / 2\right)\right] \leqslant \min \left[(1+a) / 2, a-A(a)+\left(K^{\prime} / 2\right)\right]$.

Thus we see that the following lemma is true. 
LEMMA 2. If $f \in Z$ and $-1 \leqslant a \leqslant x_{f} \leqslant b \leqslant 1$, then $\alpha_{f} \leqslant K_{1}$, in which

$$
\begin{aligned}
K_{1}=\max [A(a)+A(b)+b-a, 2 A(b) & +2 b-a-1, \\
2 A(a) & -2 a+b-1,2 A(a), 2 A(b)] .
\end{aligned}
$$

Sokolova [3] had already proved that

$$
\alpha_{f} \leqslant K_{2}=2 \min \{A(a), A(b)\}+2(b-a)
$$

if $f \in Z, a \leqslant x_{f} \leqslant b$, and $-1 \leqslant 2 a-b \leqslant 2 b-a \leqslant 1$. (This result follows easily from (4) when $\xi=a$ and $\xi=b$.) Under these circumstances it is easy to see that $K_{1} \leqslant K_{2}$ if, and only if,

$$
\Lambda \equiv|A(b)-A(a)| /(b-a) \leqslant 1,
$$

and then $K_{1}=A(a)+A(b)+b-a$. Thus we have proved the following corollary to Lemma 2.

CoRollary. Suppose that $-1 \leqslant 2 a-b<2 b-a \leqslant 1$. If $f \in Z$, if $a \leqslant x_{f} \leqslant b$, and if $\Lambda \leqslant 1$, then

$$
\alpha_{f} \leqslant K_{1}=A(a)+A(b)+b-a .
$$

It follows from Lemma 2, but not from the corollary, when $a=0, b=1$, and when $a=-1, b=0$, that $\alpha_{f} \leqslant 3 / 2$ (because $A(0)=1 / 2, A( \pm 1)=0$ ). Therefore, the set $Z$ is uniformly bounded, and $K \leqslant 3 / 2$.

5. The Second Basic Inequality. Sokolova [3] asserted that the set $Z$ is compact in the space of continuous functions on $I$. (She offered no proof, but the result is an immediate consequence of Ascoli's theorem [2], the fact that $Z$ is uniformly bounded, and the existence of a modulus of continuity [6] that applies uniformly for $f$ in $Z$, so that the functions in $Z$ are equicontinuous.) It follows that there exists a function $f_{0}$ in $Z$ and a point $x_{0}$ in $I$ such that $f_{0}\left(x_{0}\right)=K$.

Given two points $a$ and $b$ in $I$ such that $a<b$, the function $f^{*}(y)$, defined so that

$$
\begin{gathered}
f_{0}(x)=\frac{(x-a) f_{0}(b)+(b-x) f_{0}(a)}{b-a}+\frac{b-a}{2} f^{*}(y), \\
y=\frac{2(x-a)}{b-a}-1,
\end{gathered}
$$

is easily seen to be in $Z$. Therefore, $\left|f^{*}(y)\right| \leqslant K$.

Because $K \leqslant 3 / 2$, there is no loss of generality in supposing that $K^{\prime} \leqslant 3 / 2$ in (5). The existence of the function $f_{1}(x)$, described near the end of Section 3, shows that $A(\xi) \geqslant 1 / 2$ if $|\xi| \leqslant 1 / 2$. The argument leading to (5) thus shows that $K=\alpha_{f_{0}} \leqslant K^{\prime}$ if $|\xi| \leqslant 1 / 2,2 A(\xi) \leqslant K^{\prime} \leqslant 3 / 2$, and

$$
\xi+A(\xi)-\left(K^{\prime} / 2\right) \leqslant x_{0} \leqslant \xi-A(\xi)+\left(K^{\prime} / 2\right) .
$$

In particular,

$$
\begin{array}{ll}
K \leqslant K^{\prime} & \text { if } a \leqslant x_{0} \leqslant a^{\prime} \equiv a-A(a)+\left(K^{\prime} / 2\right), \\
K \leqslant K^{\prime} & \text { if } b+A(b)-\left(K^{\prime} / 2\right) \equiv b^{\prime} \leqslant x_{0} \leqslant b
\end{array}
$$


and if $2 \max \{A(a), A(b)\} \leqslant K^{\prime} \leqslant 3 / 2,0 \leqslant a<b \leqslant 1 / 2$. Note that in this case both $2 a-b$ and $b-2 a$ are in $I$.

If $a^{\prime}<b^{\prime}$, so that $K^{\prime}$ is less than the right-hand side of (6), it does not follow from (8) that $K \leqslant K^{\prime}$ if $a^{\prime} \leqslant x_{0} \leqslant b^{\prime}$. It does, however, follow from (7) that, if $a^{\prime} \leqslant x_{0} \leqslant b^{\prime}$,

$$
K \leqslant 2 \max _{a^{\prime} \leqslant x \leqslant b^{\prime}}\left\{\frac{(x-a) \Lambda(b)+(b-x) A(a)}{b-a}\right\}+\frac{(b-a) K}{2} .
$$

The maximum in (9) occurs when $x=b^{\prime}$ if $A(b) \geqslant A(a)$ and when $x=a^{\prime}$ if $A(a) \geqslant A(b)$, so that

$$
K \leqslant 2(1+\Lambda) \max \{A(a), A(b)\}-\Lambda K^{\prime}+(b-a) K / 2 .
$$

Therefore, $K$ is surely not greater than $K^{\prime}$ when $a^{\prime} \leqslant x_{0} \leqslant b^{\prime}$, if $K^{\prime}$ is not less than the value $K_{3}$ at which (10) is an equality when $K^{\prime}=K=K_{3}$. Thus we have proved the following lemma when $K_{3}<K_{1}$ and $K_{3}<3 / 2$. (Its validity is obvious if $K_{3} \geqslant K_{1}$ or $K_{3} \geqslant 3 / 2$.)

LEMmA 3. If $0 \leqslant a \leqslant x_{0} \leqslant b \leqslant 1 / 2$ and $\Lambda \leqslant 1$, then

$$
K \leqslant K_{3}=2 \max \{A(a), A(b)\} /[1-(b-a) / 2(1+\Lambda)] .
$$

The result in Lernma 3 is an improvement over an earlier result of Sokolova [3] that

$$
K \leqslant K_{4}=2 \max \{A(a), A(b)\} /[1-(b-a) / 2]
$$

if $a \leqslant x_{0} \leqslant b$. We will refer to (6) and (11) as the basic inequalities.

6. Application of the Basic Inequalities. We arrange the values of $x$, for which we exhibited upper bounds on $Z$ for $|f(x)|$ in (2), into an increasing sequence $\xi_{0}=0$, $\xi_{1}=3 / 20, \ldots, \xi_{26}=7 / 20, \xi_{27}=1$, and calculate $K_{1}$ for each interval $(a, b)=$ $\left(\xi_{i}, \xi_{i+1}\right)$, verifying along the way that $\Lambda<1$. It is obvious that $-1 \leqslant 2 a-b \leqslant 2 b$ $-a \leqslant 1$, so that the corollary to Lemma 1 is applicable, except when $(a, b)=$ $\left(\xi_{26}, \xi_{27}\right)=(7 / 20,1)$ when we must use Lemma 1 itself. The values of $K_{1}$ are recorded in Table I as the smallest six-place decimal fraction that is not less than the actual rational number computed from (6). We conclude that

$$
K \leqslant 3004633 / 2304324<1.303911 \text {. }
$$

We now calculate $K_{3}$ for each interval $(a, b)=\left(\xi_{i}, \xi_{i+1}\right)$, starting with the interval for which $K_{1}$ is largest and continuing in descending order of $K_{1}$ until we reach a value $K_{1}$ that is less than the largest value of $K_{3}$ so far calculated. In this manner we conclude that

$$
K \leqslant 1014 / 779<1.301669 \text {. }
$$

This completes the proof of the assertion that $1.3 \leqslant K<1.301669$. The estimate $K=1.300834$ is in error by less than $0.0642 \%$. 


\section{TABLE I}

Values of $K_{1}$ and $K_{3}$ calculated from the basic inequalities (6) and (11)

\begin{tabular}{|c|c|c|c|}
\hline$\xi$ & $A(\xi)$ & $K_{1}$ & $K_{3}$ \\
\hline \multirow[t]{2}{*}{0} & $1 / 2$ & & \\
\hline & & 1.293334 & \\
\hline \multirow[t]{2}{*}{$3 / 20$} & $193 / 300$ & & \\
\hline & & 1.295417 & \\
\hline \multirow[t]{2}{*}{$5 / 32$} & $31 / 48$ & & \\
\hline & $622 / 961$ & 1.298117 & \\
\hline $5 / 31$ & & 1.300767 & \\
\hline \multirow[t]{2}{*}{$1 / 6$} & $35 / 54$ & & \\
\hline & & 1.303788 & 1.301348 \\
\hline \multirow[t]{2}{*}{$4 / 23$} & $343 / 529$ & & \\
\hline & & 1.303911 & 1.301469 \\
\hline $2 / 11$ & $2821 / 4356$ & & \\
\hline \multirow[t]{2}{*}{$3 / 16$} & $373 / 576$ & 1.300864 & \\
\hline & & 1.300070 & 1.300201 \\
\hline $1 / 5$ & $16 / 25$ & & \\
\hline \multirow[t]{2}{*}{$2 / 9$} & $415 / 648$ & 1.302655 & 1.294979 \\
\hline & & 1.293210 & \\
\hline \multirow[t]{2}{*}{$1 / 4$} & $5 / 8$ & & \\
\hline & & 1.299383 & \\
\hline \multirow[t]{2}{*}{$5 / 18$} & $419 / 648$ & & \\
\hline & & 1.302501 & 1.300327 \\
\hline \multirow[t]{2}{*}{$2 / 7$} & $127 / 196$ & & \\
\hline & $161 / 248$ & 1.301848 & 1.300752 \\
\hline $9 / 31$ & & 1.302643 & 1.301511 \\
\hline \multirow[t]{2}{*}{$5 / 17$} & $751 / 1156$ & & \\
\hline & & 1.302314 & 1.301451 \\
\hline $19 / 64$ & $1331 / 2048$ & 1.300590 & \\
\hline \multirow[t]{2}{*}{$25 / 84$} & $2293 / 3528$ & & \\
\hline & & 1.302325 & 1.301514 \\
\hline $3 / 10$ & $13 / 20$ & & \\
\hline \multirow[t]{2}{*}{$23 / 76$} & $1877 / 2888$ & 1.302563 & 1.301669 \\
\hline & & 1.302547 & 1.301621 \\
\hline \multirow[t]{2}{*}{$11 / 36$} & $421 / 648$ & & \\
\hline & & 1.302753 & 1.301627 \\
\hline \multirow[t]{2}{*}{$13 / 42$} & $1145 / 1764$ & & \\
\hline & & 1.302631 & 1.301090 \\
\hline \multirow[t]{2}{*}{$11 / 35$} & $3179 / 4900$ & & \\
\hline & $313 / 484$ & 1.299366 & \\
\hline $7 / 22$ & & 1.300735 & \\
\hline \multirow[t]{2}{*}{$1 / 3$} & $23 / 36$ & & \\
\hline & & 1.290556 & \\
\hline \multirow[t]{2}{*}{$7 / 20$} & $127 / 200$ & & \\
\hline & 0 & 1.285000 & \\
\hline
\end{tabular}

7. A Related Linear Programming Problem. Although our paper is logically complete, it may be helpful to explain the origin of the various identities used in Section 2 to obtain upper bounds, and of the various piecewise linear functions in $Z$ that were (or could have been) used in Section 3 to obtain lower bounds. 
We say that the $2 N+1^{\prime}$ tuple $y=\left(y_{0}, y_{1}, y_{2}, \ldots, y_{2 N-1}, y_{2 N}\right)$ is admissible if $y_{0}=y_{2 N}=0$ and the inequalities (3) hold whenever $k$ and $l$ are integers such that $0 \leqslant k \leqslant 2 N-2,1 \leqslant l \leqslant N-(k / 2)$. Let $P_{m N}$ be the (linear programming) problem of finding an admissible $y$ for which $y_{m}$ is a maximum. Let $y_{m N}$ be this maximum value. Then $y_{m N}=y_{2 N-m, N}$ because $f(-x) \in Z$ when $f(x) \in Z$. It is obvious that $f\left(x_{m}\right) \leqslant y_{m N}$ if $f \in Z$.

We say that the $2 N+1^{\prime}$ tuple $y$ is *admissible if $y_{0}=y_{2 N}=0$ and the inequalities (3) hold whenever $k$ and $2 l$ are integers such that $0 \leqslant k \leqslant 2 N-2,2 \leqslant 2 l \leqslant 2 N-k$ and the value $y_{k+1 / 2}$ is defined as $\left(y_{k}+y_{k+1}\right) / 2$. Let $P_{m N}^{*}$ be the linear programming problem of finding a *admissible $y$ for which $y_{m}$ is a maximum. Let $y_{m N}^{*}$ be this maximum value. By virtue of Lemma 1 it is obvious that

$$
\sup _{f \in Z} f\left(x_{m}\right) \geqslant y_{m N}^{*} \text {. }
$$

We have written a computing program for the Cyber-176 that calculates the coefficients for both $P_{m N}$ and $P_{m N}^{*}$, and stores them for use in the general purpose linear programming routine APEX III [8]. Each of the points $\xi$, for which an upper bound for $|f(\xi)|$ was furnished in (2), is a rational fraction of the form $x_{m}=$ $(m-N) / N$ in which $m$ and $N$ are relatively prime integers and $N \leqslant m<2 N$. Accordingly, we can use the computer to find $y_{m N}$ and $y_{m N}^{*}$.

The computer output for $P_{m N}$ contains, among other things, not only $y_{m N}$ and the values $y_{k}(k=1,2, \ldots, 2 N)$ corresponding to the maximizing solution, but also the values of the "marginal" variables ( $=$ dual variables $=$ Lagrange multipliers) corresponding to each of the $2 N^{2}$ inequalities (3). It is a well-known fact that the sum of the products of each marginal variable and the left-hand side of the corresponding inequality is identically equal to the function being maximized. These identities are the ones recorded in the appendix at the end of the paper after two modifications. All of the computer output is expressed as finite decimal fractions (mostly 5 place accuracy). We have guessed simple rational fractions to which the decimal fraction values for the marginal variables appeared to be reasonably accurate approximations. That our guesses were appropriate is confirmed by the observations that the identities in the appendix, written down with the guessed rational fractions (and multiplied by a least common denominator in order to use only integers as coefficients), are actually identities, verifiable ab initio without any refernce to their computer origin. Next, the integers $m$ and $N$ can be multiplied by the same factor $\kappa$ without changing $x_{m}$. It is obvious that $y_{\kappa m, \kappa N} \leqslant y_{m N}$ and $y_{\kappa m, \kappa N}^{*} \geqslant y_{m N}^{*}$ if $\kappa$ is a positive integer. Accordingly, the identity actually recorded in the appendix corresponds to the smallest $\kappa$ in the set of $\kappa$ for which the problem $P_{\kappa m, \kappa N}$ can be handled by APEX-III on our computer and for which $y_{\kappa m, \kappa N}$ is least. It may happen that there is an integer $\kappa^{\prime}$ for which the problem $P_{\kappa^{\prime} m . \kappa^{\prime} N}^{*}$ can be handled by APEX-III on our computer and for which $y_{\kappa^{\prime} m, \kappa^{\prime} N}^{*}$ is equal to the least $y_{\kappa m, \kappa N}$. In this case,

$$
\sup _{f \in Z} f\left(x_{m}\right)=y_{\kappa m, \kappa N},
$$

so there is nothing to be gained by proceeding to larger values of $\boldsymbol{\kappa}$ or $\boldsymbol{\kappa}^{\prime}$. The numerical data show that it is possible that $y_{2 m, 2 N}=y_{m, N}$ (e.g., $y_{12,8}=y_{6,4}=y_{3,2}=1$ ), that $y_{2 m, 2 N}<y_{m, N}$ (e.g., $\left.y_{38,32}=83 / 64<y_{19,16}=13 / 10\right)$, that $y_{m N}^{*}=y_{m N}$ (e.g., $y_{5,4}^{*}$ $\left.=y_{5,4}=5 / 4\right)$, that $y_{m N}^{*}<y_{m N}\left(\right.$ e.g., $\left.y_{7,6}^{*}=23 / 18<y_{7,6}=35 / 27\right)$, that $\kappa^{\prime}=\kappa>1$ 
(e.g., $y_{17,13}^{*}<y_{34,26}^{*}<y_{51.39}^{*}<y_{68.52}^{*}=439 / 338=y_{68.52}<y_{34.26}<y_{51,39}<y_{17.13}$ ), that $\kappa^{\prime}<\kappa$ (e.g., $y_{11,9}^{*}<y_{22,18}^{*}<y_{33,27}^{*}<y_{44.36}^{*}=415 / 324=y_{88,72}<y_{77.63}<y_{66.54}<y_{55.45}$ $\left.<y_{44,36}<y_{33,27}=y_{22,18}=y_{11,9}\right)$, and that $\kappa^{\prime}>\kappa\left(\right.$ e.g., $y_{23,20}^{*}<y_{46,40}^{*}<y_{69.60}^{*}=$ $\left.193 / 150=y_{46.40}<y_{23.20}\right)$. The fifth of these examples also shows that $y_{\kappa m, \kappa N}$ need not be monotone in $\kappa$.

The computer output for $P_{m N}^{*}$ contains not only $y_{m N}^{*}$ but also the values $y_{k}^{*}$ $(k=1, \ldots, 2 N-1)$ corresponding to the maximizing solutions. These decimal fractions satisfy conditions (3) as in Lemma 1, within some unknown round-off error. We have replaced the decimal fractions with simple rational fractions to which the decimal fraction appeared to be reasonably accurate approximations. The new values of $y_{k}^{*}$, recorded in the case $N=20, m=26$ and alluded to in other cases in Section 3 , were then independently verified to satisfy (3) without any reference to their computer origin. This verification can be carried out by arithmetical operations in the ring of integers if each $y_{k}^{*}$ and the tests (3) are first multiplied by the least common denominator of the numbers $y_{k}^{*}$. No round-off errors need occur and the arithmetic can be programmed for our computer.

A Set of Useful Identities

$$
\begin{aligned}
& 64 f(19 / 64)=8[2 f(19 / 64)-f(-13 / 32)-f(1)]+8[2 f(19 / 64) \\
& -f(17 / 64)-f(21 / 64)]+10[2 f(19 / 64)-f(3 / 32) \\
& -f(1 / 2)]+4[2 f(19 / 64)-f(7 / 64)-f(31 / 64)] \\
& +2[2 f(19 / 64)-f(9 / 64)-f(29 / 64)]+4[2 f(-13 / 32) \\
& -f(-33 / 64)-f(-19 / 64)]+4[2 f(17 / 64)-f(1 / 32) \\
& -f(1 / 2)]+4[2 f(21 / 64)-f(-11 / 32)-f(1)] \\
& +10[f(-3 / 32)+f(3 / 32)-2 f(0)]+4[f(-19 / 64) \\
& +f(7 / 64)-2 f(-3 / 32)]+2[2 f(31 / 64)-f(-1 / 32) \\
& -f(1)]+2[f(-9 / 64)+f(9 / 64)-2 f(0)] \\
& +[2 f(29 / 64)-f(-3 / 32)-f(1)]+2[2 f(-33 / 64) \\
& -f(-1)-f(-1 / 32)]+4[f(-1 / 32)+f(1 / 32) \\
& -2 f(0)]+2[2 f(-11 / 32)-f(-35 / 64)-f(-9 / 64)] \\
& +[2 f(-35 / 64)-f(-1)-f(-3 / 32)]+3 f(-1) \\
& +32 f(0)+14 f(1 / 2)+15 f(1) \text {, } \\
& 76 f(23 / 76)=20[2 f(23 / 76)-f(21 / 76)-f(25 / 76)]+8[2 f(23 / 76) \\
& -f(3 / 19)-f(17 / 38)]+4[2 f(23 / 76)-f(2 / 19) \\
& -f(1 / 2)]+6[2 f(23 / 76)-f(-15 / 38)-f(1)] \\
& +10[2 f(21 / 76)-f(1 / 19)-f(1 / 2)]+10[2 f(25 / 76) \\
& -f(-13 / 38)-f(1)]+3[f(-5 / 19)+f(3 / 19) \\
& -2 f(-1 / 19)]+5[f(-3 / 19)+f(3 / 19)-2 f(0)] \\
& +4[2 f(17 / 38)-f(-2 / 19)-f(1)]+4[f(-2 / 19) \\
& +f(2 / 19)-2 f(0)]+3[2 f(-15 / 38)-f(-10 / 19)
\end{aligned}
$$


$-f(-5 / 19)]+10[f(-1 / 19)+f(1 / 19)-2 f(0)]$

$+5[2 f(-13 / 38)-f(-10 / 19)-f(-3 / 19)]+4[2 f(-10 / 19)$

$-f(-1)-f(-1 / 19)]+4 f(-1)+38 f(0)$

$+14 f(1 / 2)+20 f(1)$,

$126 f(25 / 84)=16[2 f(25 / 84)-f(11 / 42)-f(1 / 3)]+16[2 f(25 / 84)$

$-f(23 / 84)-f(9 / 28)]+16[2 f(25 / 84)-f(2 / 7)$

$-f(13 / 42)]+5[2 f(25 / 84)-f(2 / 21)-f(1 / 2)]$

$+4[2 f(25 / 84)-f(5 / 42)-f(10 / 21)]+4[2 f(25 / 84)$

$-f(11 / 84)-f(13 / 28)]+2[2 f(25 / 84)-f(1 / 7)$

$-f(19 / 42)]+8[2 f(11 / 42)-f(1 / 42)-f(1 / 2)]$

$+8[2 f(1 / 3)-f(19 / 84)-f(37 / 84)]+8[2 f(23 / 84)$

$-f(1 / 21)-f(1 / 2)]+8[2 f(9 / 28)-f(-5 / 14)$

$-f(1)]+8[2 f(2 / 7)-f(1 / 14)-f(1 / 2)]$

$+3[2 f(13 / 42)-f(5 / 21)-f(8 / 21)]+2[f(-5 / 21)$

$+f(2 / 21)-2 f(-1 / 14)]+3[f(-2 / 21)+f(2 / 21)$

$-2 f(0)]+4[f(-5 / 42)+f(5 / 42)-2 f(0)]$

$+2[2 f(10 / 21)-f(-1 / 21)-f(1)]+4[f(-5 / 28)$

$+f(11 / 84)-2 f(-1 / 42)]+2[2 f(13 / 28)-f(-1 / 14)$

$-f(1)]+2[f(-5 / 21)+f(1 / 7)-2 f(-1 / 21)]$

$+3[2 f(19 / 42)-f(-2 / 21)-f(1)]+4[2 f(19 / 84)$

$-f(0)-f(19 / 42)]+8[f(-1 / 42)+f(1 / 42)$

$-2 f(0)]+4[2 f(37 / 84)-f(-5 / 42)-f(1)]$

$+8[f(-1 / 21)+f(1 / 21)-2 f(0)]+4[2 f(-5 / 14)$

$-f(-15 / 28)-f(-5 / 28)]+8[f(-1 / 14)+f(1 / 14)$

$-2 f(0)]+4[2 f(5 / 21)-f(-11 / 21)-f(1)]$

$+4[2 f(8 / 21)-f(-5 / 21)-f(1)]+2[2 f(-15 / 28)$

$-f(-1)-f(-1 / 14)]+2[2 f(-11 / 21)-f(-1)$

$-f(-1 / 21)]+4 f(-1)+66 f(0)+29 f(1 / 2)+27 f(1)$.

$\therefore 1111[\because(1 / 4)-f(0)-f(1 / 2)]+f(0)+f(1 / 2)$,

bif $(1 / 3) \quad \therefore[2 f(1 / 3)-f(-1 / 3)-f(1)]+2 f(1)$

$+[2 f(1 / 3)-f(1 / 6)-f(1 / 2)]+f(1 / 2)$

$+[2 f(-1 / 3)-f(-1 / 6)-f(-1 / 2)]+f(-1 / 2)$

$+[f(-1 / 6)+f(1 / 6)-2 f(0)]+2 f(0)$,

$5 f(1 / 5)=2[2 f(1 / 5)-f(-3 / 5)-f(1)]+2 f(1)$

$+[f(-1 / 5)+f(1 / 5)-2 f(0)]+2 f(0)$

$+[2 f(-3 / 5)-f(-1)-f(-1 / 5)]+f(-1)$, 


$$
\begin{aligned}
& 9 f(1 / 6)=4[2 f(1 / 6)-f(-2 / 3)-f(1)]+4 f(1) \\
& +[f(-1 / 6)+f(1 / 6)-2 f(0)]+2 f(0) \\
& +2[2 f(-2 / 3)-f(-1)-f(-1 / 3)]+2 f(-1) \\
& +[2 f(-1 / 3)-f(-1 / 2)-f(-1 / 6)]+f(-1 / 2) \text {, } \\
& 14 f(2 / 7)=3[2 f(2 / 7)-f(1 / 14)-f(1 / 2)]+4[2 f(2 / 7) \\
& -f(-3 / 7)-f(1)]+[f(-3 / 14)-2 f(-1 / 14) \\
& +f(1 / 14)]+2[f(-1 / 14)-2 f(0)+f(1 / 14)] \\
& +2[2 f(-3 / 7)-f(-1 / 2)-f(-5 / 14)]+[2 f(-5 / 14) \\
& -f(-1 / 2)-f(-3 / 14)]+3 f(1 / 2)+4 f(1) \\
& +4 f(0)+3 f(-1 / 2) \text {, } \\
& 36 f(2 / 9)=10[2 f(2 / 9)-f(0)-f(4 / 9)]+4[2 f(2 / 9) \\
& -f(5 / 24)-f(17 / 72)]+4[2 f(2 / 9)-f(7 / 36) \\
& -f(1 / 4)]+5[2 f(4 / 9)-f(-1 / 9)-f(1)] \\
& +2[2 f(5 / 24)-f(1 / 9)-f(11 / 36)]+2[2 f(17 / 72) \\
& -f(0)-f(17 / 36)]+2[2 f(7 / 36)-f(1 / 9) \\
& -f(5 / 18)]+5[f(-1 / 9)+f(1 / 9)-2 f(0)] \\
& +[2 f(11 / 36)-f(1 / 9)-f(1 / 2)]+[2 f(17 / 36) \\
& -f(-1 / 18)-f(1)]+[2 f(5 / 18)-f(1 / 10) \\
& -f(1 / 2)]+[f(-1 / 18)+f(1 / 18)-2 f(0)] \\
& +24 f(0)+4 f(1 / 4)+2 f(1 / 2)+6 f(1) \text {, } \\
& 264 f(2 / 11)=28[2 f(2 / 11)-f(-7 / 11)-f(1)]+104[2 f(2 / 11) \\
& -f(5 / 33)-f(7 / 33)]+4[2 f(5 / 33)-f(1 / 33) \\
& -f(3 / 11)]+14[f(-3 / 11)-2 f(-2 / 33)+f(5 / 33)] \\
& +28[2 f(5 / 33)-f(2 / 33)-f(8 / 33)]+26[f(5 / 33) \\
& -2 f(0)+f(-5 / 33)]+52[2 f(7 / 33)-f(-19 / 33) \\
& -f(1)]+14[2 f(-7 / 11)-f(-1)-f(-3 / 11)] \\
& +2[2 f(3 / 11)-f(1 / 33)-f(17 / 33)]+7[f(-1 / 33) \\
& -2 f(0)+f(1 / 33)]+[2 f(17 / 33)-f(1 / 33) \\
& -f(1)]+28[f(-2 / 33)-2 f(0)+f(2 / 33)] \\
& +14[2 f(8 / 33)-f(0)-f(16 / 33)]+7[2 f(16 / 33) \\
& -f(-1 / 33)-f(1)]+25[2 f(-19 / 33)-f(-1) \\
& -f(-5 / 33)]+136 f(0)+88 f(1)+40 f(-1) \text {, } \\
& 10 f(3 / 10)=4[2 f(3 / 10)-f(-2 / 5)-f(1)]+4 f(1) \\
& +[2 f(3 / 10)-f(1 / 10)-f(1 / 2)]+f(1 / 2) \\
& +2[2 f(-2 / 5)-f(-1 / 2)-f(-/ / 10)]+3 f(-1 / 2)
\end{aligned}
$$




$$
\begin{aligned}
& +[f(-1 / 10)-2 f(0)+f(1 / 10)]+2 f(0) \\
& +[2 f(-3 / 10)-f(-1 / 2)-f(-1 / 10)], \\
& 24 f(3 / 16)=8[2 f(3 / 16)-f(1 / 6)-f(5 / 24)]+12[2 f(3 / 16) \\
& -f(-1 / 48)-f(19 / 48)]+2[2 f(3 / 16)-f(0) \\
& -f(3 / 8)]+2[2 f(1 / 6)-f(1 / 12)-f(1 / 4)] \\
& +[f(-1 / 4)+f(1 / 6)-2 f(-1 / 24)]+[f(-5 / 24) \\
& +f(1 / 6)-2 f(-1 / 48)]+2[f(-1 / 6)+f(1 / 6) \\
& -2 f(0)]+4[2 f(5 / 24)-f(0)-f(5 / 12)] \\
& +4[f(-1 / 48)+f(1 / 48)-2 f(0)]+[2 f(19 / 48) \\
& -f(-5 / 24)-f(1)]+[2 f(3 / 8)-f(-1 / 4) \\
& -f(1)]+2[f(-1 / 24)+f(1 / 12)-2 f(1 / 48)] \\
& +2[2 f(5 / 12)-f(-1 / 6)-f(1)]+18 f(0) \\
& +2 f(1 / 4)+4 f(1) \text {, } \\
& 60 f(3 / 20)=14[2 f(3 / 20)-f(1 / 20)-f(1 / 4)]+12[2 f(3 / 20) \\
& \text { - } f(1 / 8)-f(7 / 40)]+4[2 f(3 / 20)-f(1 / 10) \\
& -f(1 / 5)]+14[f(-1 / 20)+f(1 / 20)-2 f(0)] \\
& +6[2 f(1 / 8)-f(-3 / 4)-f(1)]+6[2 f(7 / 40) \\
& -f(-1 / 20)-f(2 / 5)]+4[f(-1 / 5)+f(1 / 10) \\
& -2 f(-1 / 20)]+2[2 f(1 / 5)-f(0)-f(2 / 5)] \\
& +3[2 f(-3 / 4)-f(-1)-f(-1 / 2)]+4[2 f(2 / 5) \\
& -f(-1 / 5)-f(1)]+3 f(-1)+3 f(-1 / 2) \\
& +30 f(0)+14 f(1 / 4)+10 f(1) \text {, } \\
& 92 f(4 / 23)=40[2 f(4 / 23)-f(3 / 23)-f(5 / 23)]+6[2 f(4 / 23) \\
& -f(-1 / 23)-f(9 / 23)]+12[2 f(3 / 23)-f(1 / 23) \\
& -f(5 / 23)]+13[f(-3 / 23)+f(3 / 23)-2 f(0)] \\
& +3[f(-5 / 23)+f(3 / 23)-2 f(-1 / 23)]+26[2 f(5 / 23) \\
& -f(0)-f(10 / 23)]+12[f(-1 / 23)+f(1 / 23) \\
& -2 f(0)]+3[2 f(9 / 23)-f(-5 / 23)-f(1)] \\
& +13[2 f(10 / 23)-f(-3 / 23)-f(1)]+76 f(0)+16 f(1) \text {, } \\
& 68 f(5 / 17)=14[2 f(5 / 17)-f(3 / 34)-f(1 / 2)]+8[2 f(5 / 17) \\
& -f(-7 / 17)-f(1)]+4[2 f(5 / 17)-f(9 / 68) \\
& -f(31 / 68)]+4[2 f(5 / 17)-f(9 / 34)-f(11 / 34)] \\
& +4[2 f(5 / 17)-f(19 / 68)-f(21 / 68)]+12[f(-3 / 34) \\
& +f(3 / 34)-2 f(0)]+[f(-7 / 34)+f(3 / 34) \\
& -2 f(-1 / 17)]+[f(-9 / 34)+f(3 / 34)-2 f(-3 / 34)]
\end{aligned}
$$




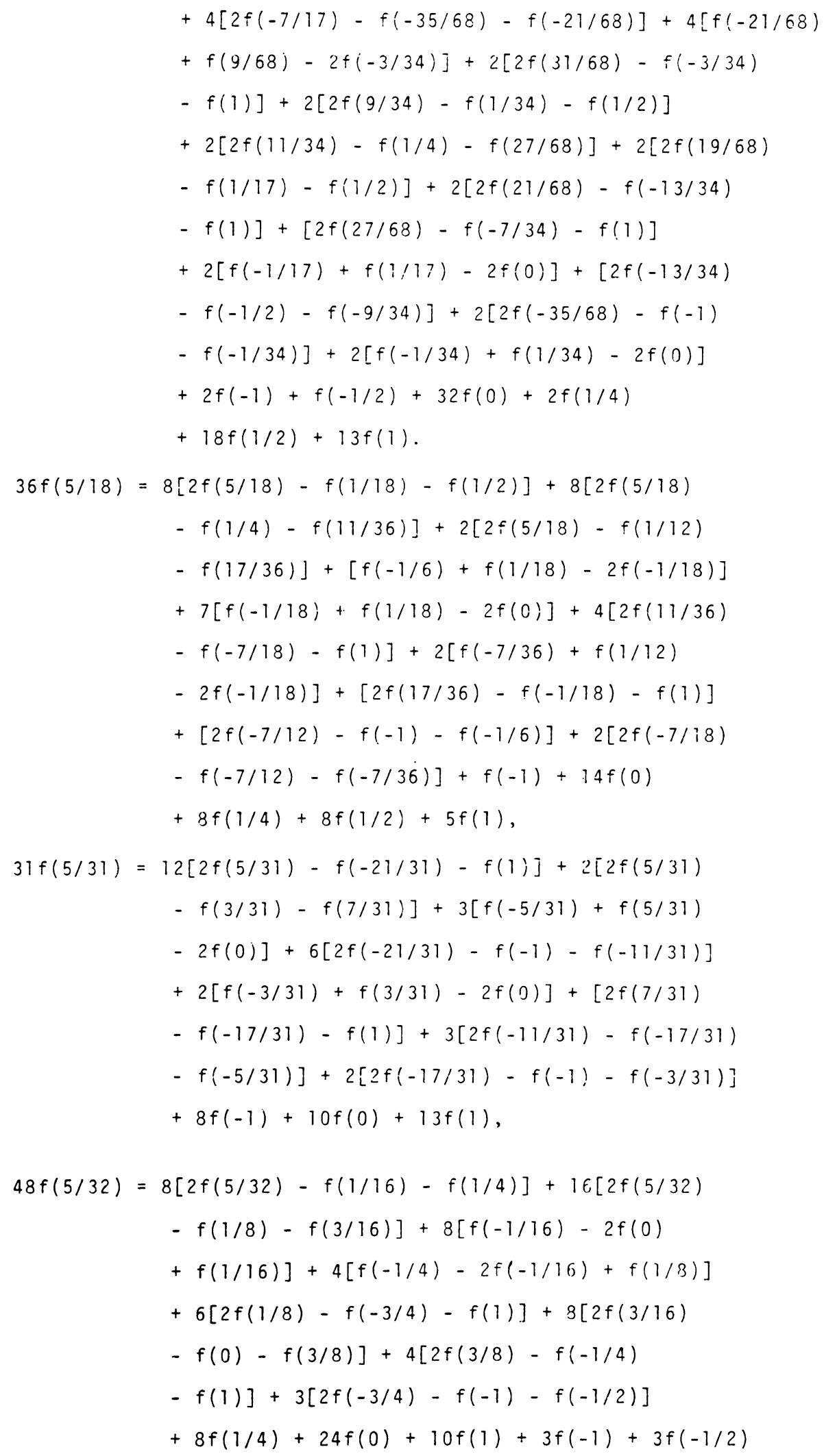




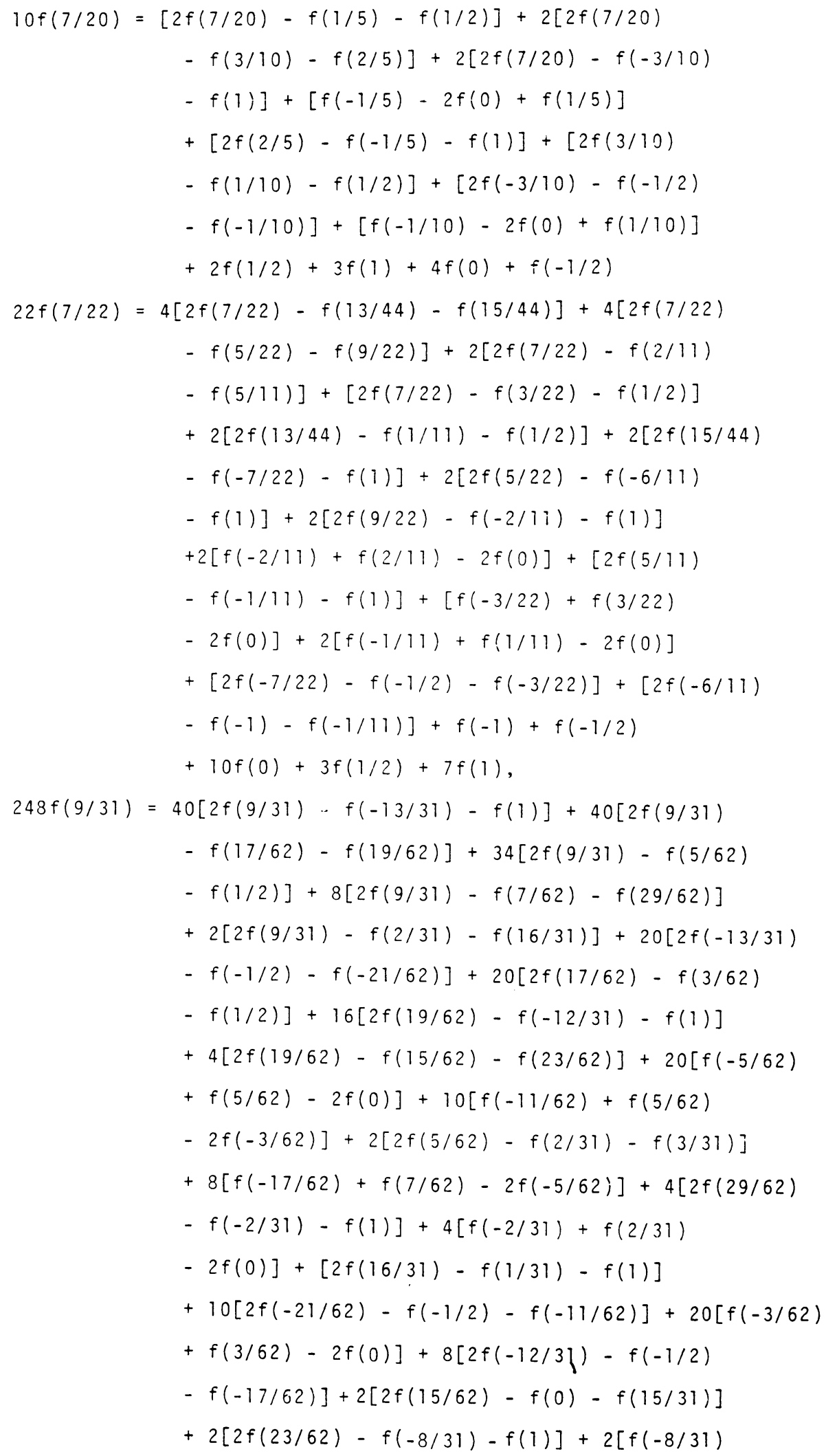




$$
\begin{aligned}
& +f(3 / 31)-2 f(-5 / 62)]+[f(-1 / 31)+f(1 / 31) \\
& -2 f(0)]+[2 f(15 / 31)-f(-1 / 31)-f(1)] \\
& +38 f(-1 / 2)+92 f(0)+54 f(1 / 2)+64 f(1) \text {, } \\
& 700 f(11 / 35)=144[2 f(11 / 35)-f(-13 / 35)-f(1)]+72[2 f(11 / 35) \\
& -f(4 / 35)-f(18 / 35)]+72[2 f(11 / 35)-f(13 / 70) \\
& -f(31 / 70)]+28[2 f(11 / 35)-f(8 / 35)-f(2 / 5)] \\
& +14[2 f(11 / 35)-f(1 / 7)-f(17 / 35)]+8[2 f(11 / 35) \\
& -f(3 / 10)-f(23 / 70)]+8[2 f(11 / 35)-f(2 / 7) \\
& -f(12 / 35)]+2[2 f(11 / 35)-f(6 / 35)-f(16 / 35)] \\
& +2[2 f(11 / 35)-f(9 / 70)-f(1 / 2)]+72[2 f(-13 / 35) \\
& -f(-39 / 70)-f(-13 / 70)]+72[f(-4 / 35)+f(4 / 35) \\
& -2 f(0)]+36[2 f(18 / 35)-f(1 / 35)-f(1)] \\
& +72[f(-13 / 70)+f(13 / 70)-2 f(0)]+36[2 f(31 / 70) \\
& -f(-4 / 35)-f(1)]+14[2 f(8 / 35)-f(-19 / 35) \\
& -f(1)]+14[f(-1 / 5)+f(1 / 7)-2 f(-1 / 35)] \\
& +14[2 f(2 / 5)-f(-1 / 5)-f(1)]+8[2 f(17 / 35) \\
& -f(-1 / 35)-f(1)]+2[2 f(17 / 70)-f(0) \\
& -f(17 / 35)]+4[2 f(3 / 10)-f(1 / 10)-f(1 / 2)] \\
& +4[2 f(23 / 70)-f(17 / 70)-f(29 / 70)]+4[2 f(2 / 7) \\
& -f(1 / 14)-f(1 / 2)]+4[2 f(12 / 35)-f(-11 / 35) \\
& -f(1)]+2[f(-6 / 35)+f(6 / 35)-2 f(0)] \\
& +[2 f(16 / 35)-f(-3 / 35)-f(1)]+2[f(-9 / 70) \\
& +f(9 / 70)-2 f(0)]+36[2 f(-39 / 70)-f(-1) \\
& -f(-4 / 35)]+36[f(-1 / 35)+f(1 / 35)-2 f(0)] \\
& +7[2 f(-19 / 35)-f(-1)-f(-3 / 35)]+4[f(1 / 14) \\
& +f(1 / 10)-2 f(3 / 35)]+8[f(-3 / 35)+f(3 / 35) \\
& -2 f(0)]+2[2 f(29 / 70)-f(-6 / 35)-f(1)] \\
& +2[2 f(-11 / 35)-f(-1 / 2)-f(-9 / 70)]+43 f(-1) \\
& +2 f(-1 / 2)+386 f(0)+10 f(1 / 2)+259 f(1) \text {, } \\
& 36 f(11 / 36)=12[2 f(11 / 36)-f(5 / 18)-f(1 / 3)]+4[2 f(11 / 36) \\
& -f(5 / 36)-f(17 / 36)]+2[2 f(11 / 36)-f(-7 / 18) \\
& -f(1)]+6[2 f(5 / 18)-f(1 / 18)-f(1 / 2)] \\
& +6[2 f(1 / 3)-f(-1 / 3)-f(1)]+3[f(-5 / 36) \\
& +f(5 / 36)-2 f(0)]+[f(-1 / 4)+f(5 / 36) \\
& -2 f(-1 / 18)]+2[2 f(17 / 36)-f(-1 / 18)-f(1)]
\end{aligned}
$$


$+[2 f(-7 / 18)-f(-19 / 36)-f(-1 / 4)]+6[f(-1 / 18)$

$+f(1 / 18)-2 f(0)]+3[2 f(-1 / 3)-f(-19 / 36)$

$-f(-5 / 36)]+2[2 f(-19 / 36)-f(-1)-f(-1 / 18)]$

$+2 f(-1)+18 f(0)+6 f(1 / 2)+10 f(1)$,

$$
\begin{aligned}
294 f(13 / 42)= & 64[2 f(13 / 42)-f(2 / 7)-f(1 / 3)]+33[2 f(13 / 42) \\
& -f(5 / 42)-f(1 / 2)]+30[2 f(13 / 42)-f(1 / 4) \\
& -f(31 / 84)]+16[2 f(13 / 42)-f(25 / 84)-f(9 / 28)] \\
& +4[2 f(13 / 42)-f(13 / 84)-f(13 / 28)]+32[2 f(2 / 7) \\
& -f(1 / 14)-f(1 / 2)]+32[2 f(1 / 3)-f(-1 / 3) \\
& -f(1)]+18[f(-5 / 42)+f(5 / 42)-2 f(0)] \\
& +15[f(-11 / 42)+f(5 / 42)-2 f(-1 / 14)]+15[2 f(31 / 84) \\
& -f(-11 / 42)-f(1)]+8[2 f(25 / 84)-f(2 / 21) \\
& -f(1 / 2)]+8[2 f(9 / 28)-f(-5 / 14)-f(1)] \\
& +4[f(-13 / 84)+f(13 / 84)-2 f(0)]+2[2 f(13 / 28) \\
& -f(-1 / 14)-f(1)]+32[f(-1 / 14)+f(1 / 14) \\
& -2 f(0)]+16[2 f(-1 / 3)-f(-23 / 42)-f(-5 / 42)] \\
& +2[2 f(-47 / 84)-f(-1)-f(-5 / 42)]+8[f(-2 / 21) \\
& +f(2 / 21)-2 f(0)]+4[2 f(-5 / 14)-f(-47 / 34) \\
& -f(-13 / 84)]+8[2 f(-23 / 42)-f(-1)-f(-2 / 21)] \\
& +10 f(-1)+124 f(0)+30 f(1 / 4)+73 f(1 / 2)+57 f(1),
\end{aligned}
$$$$
64 f(19 / 64)=8[2 f(19 / 64)-f(-13 / 32)-f(1)]+8[2 f(19 / 64)
$$$$
-f(17 / 64)-f(21 / 64)]+10[2 f(19 / 64)-f(3 / 32)
$$$$
-f(1 / 2)]+4[2 f(19 / 64)-f(7 / 64)-f(31 / 64)]
$$$$
+2[2 f(19 / 64)-f(9 / 64)-f(29 / 64)]+4[2 f(-13 / 32)
$$$$
-f(-33 / 64)-f(-19 / 64)]+4[2 f(17 / 64)-f(1 / 32)
$$$$
-f(1 / 2)]+4[2 f(21 / 64)-f(-11 / 32)-f(1)]
$$$$
+10[f(-3 / 32)+f(3 / 32)-2 f(0)]+4[f(-19 / 64)
$$$$
+f(7 / 64)-2 f(-3 / 32)]+2[2 f(31 / 64)-f(-1 / 32)
$$$$
-f(1)]+2[f(-9 / 64)+f(9 / 64)-2 f(0)]
$$$$
+[2 f(29 / 64)-f(-3 / 32)-f(1)]+2[2 f(-33 / 64)
$$$$
-f(-1)-f(-1 / 32)]+4[f(-1 / 32)+f(1 / 32)
$$$$
-2 f(0)]+2[2 f(-11 / 32)-f(-35 / 64)-f(-9 / 64)]
$$$$
+[2 f(-35 / 64)-f(-1)-f(-3 / 32)]+3 f(-1)
$$$$
+32 f(0)+14 f(1 / 2)+15 f(1) \text {, }
$$ 


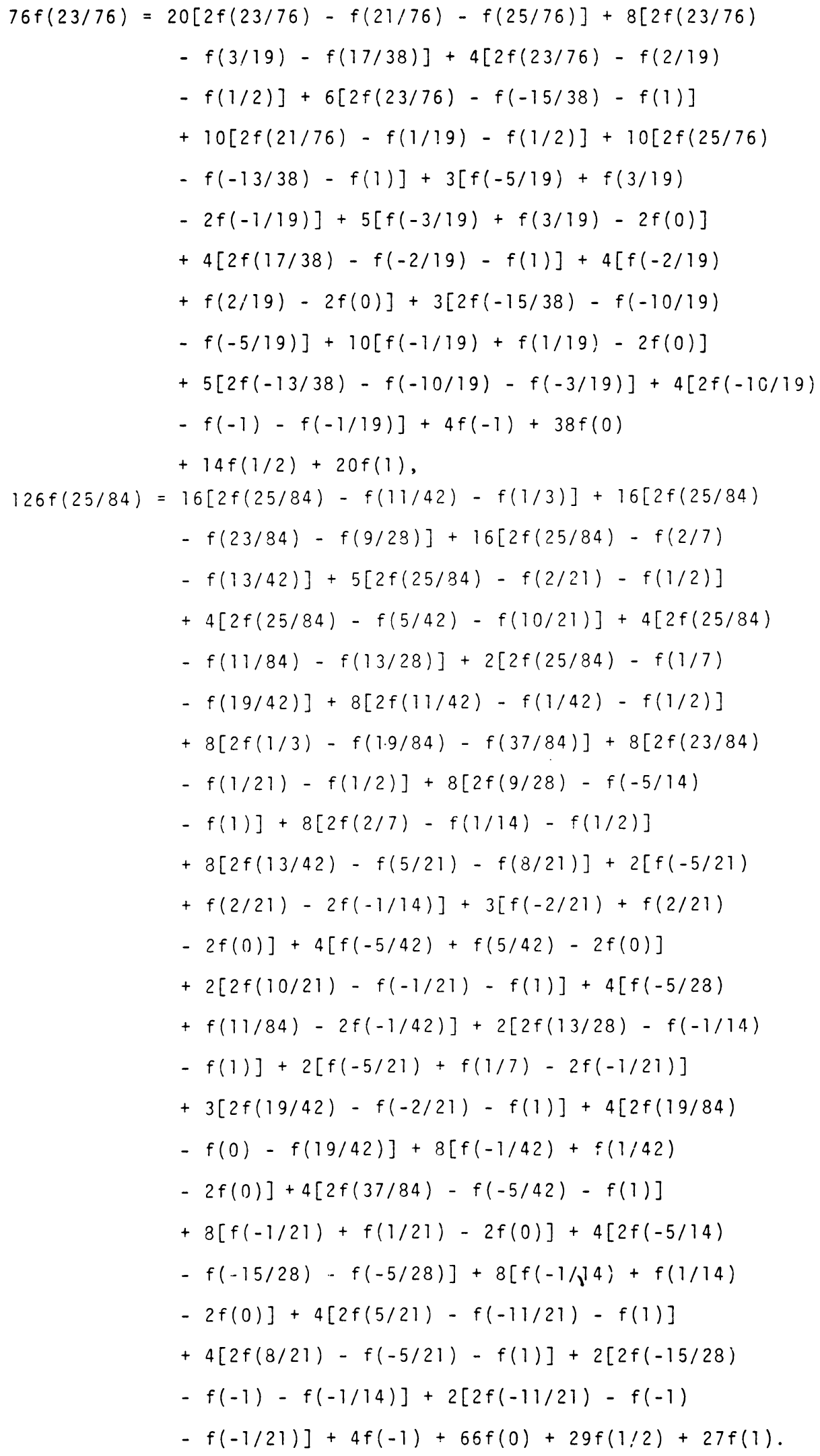


EG \& G Idaho, Inc.

P. O. Box 1625

Idaho Falls, Idaho 83415

1. YU. A. Brudnyi, "On the maximum modulus of a quasi-smooth function," Uspehi Mat. Nauk ( N. S.), v. 12, no. 4(76), 1957, pp. 273-275. (Russian) MR 19. 733.

2. L. M. Graves, The Theory of Functions of Real Variables, McGraw-Hill, New York, 1946, p. 122. MR 8, 319.

3. 1. P. Sokolova, "The maximum of the modulus of a function that satisfies Zygmund's condition," Studies in the Theory of Functions of Several Real Variables, Jaroslav. Gos. Univ., Yaroslavl, 1976, pp. 66-71. (Russian) MR 58 \#28343.

4. A. F. Timan, "Quasi-smooth functions,” Dokl. Akad. Nauk SSSR (N.S.). v. 70, 1950, pp. 961-963. (Russian) MR 11, 422.

5. A. F. Timan, "Quasi-smooth functions," Uspehi Mat. Nauk (N.S.), v. 5, no. 3(37). 1950. pp. 128-130. (Russian) MR 12, 13.

6. A. F. TIman, "On quasi-smooth functions," Izt. Akad. Nauk SSSR Ser. Mat., v. 15. 1951, pp. 243-254. (Russian) MR 13, 17.

7. A. Zygmund, "Smooth functions," Duke Math. J., v. 12, 1945, pp. 47-76. MR 7, 60.

8. APEX-III Reference Manual, Version 1.2, Control Data Corporation Manual \#76070000, March 12, 1979. 